Pensamiento Crítico Vol. 19 № 2, pp. 21-37

\title{
Sin armonía no habrá crecimiento sostenido
}

\author{
Disharmonious will be no sustained growth
}

Mag. Jorge Barrera Herrera*

\section{RESUMEN}

La tradicional y marcada diferencia entre los países ricos del norte y los países pobres del sur, desde aproximadamente 1990 está desapareciendo, cada vez más. Esa marcada divergencia entre los cada vez países más ricos y los cada vez países más pobres, que se inició con la revolución industrial y el colonialismo a inicios del siglo XIX, se está revirtiendo por una nueva convergencia.

Hoy en día se está produciendo un cambio estructural en la dinámica de la economía mundial, además de esta nueva convergencia por el comportamiento acelerado de la tasa de crecimiento económica per cápita de los países emergentes y en desarrollo que es mayor a la de los países desarrollados y que tiende a homogeneizar los crecimientos per cápita globales, sin embargo estos países no se han podido desvincular de la fuerte correlación cíclica en torno a la tendencia y tampoco se han podido aislar de los efectos de la creciente divergencia en la desigualdad en la distribución de los ingresos entre ricos y pobres.

* Profesor asociado y miembro investigador del Instituto de Investigaciones de la Facultad de Ciencias Económicas de la UNMSM. Estudios de doctorado en Economía UNMSM. Magíster en Administración UESAN. Licenciado en Ciencias Marítimas Navales ESNA. 


\section{Pensamiento Crítico Vol. 19. № 2}

Las 85 personas más ricas del mundo poseen ahora más de la mitad de la riqueza disponible, la desigualdad en la distribución del ingreso está divergiendo, potenciándose y perpetuándose a un ritmo sin precedentes a nivel mundial, (a pesar de que el ingreso promedio global está convergiendo), dejando una impresión cada vez mayor de que es "moralmente incorrecta", además está demostrado que frena la velocidad de crecimiento de los países.

A pesar de que en este artículo nos centramos en la desigualdad del ingreso global, esta a menudo está asociada a otras desigualdades que también afectan globalmente y podrían eventualmente ser más importantes aún (carencia en el acceso a los alimentos, al agua, la sanidad, la educación, etc.).

La propiedad del capital está más concentrada que la distribución de las rentas; el $10 \%$ de la población de la OCDE tienen más del $50 \%$ de la propiedad, por otra parte un $50 \%$ de la población no tiene ninguna propiedad, pero si está endeudada.

Palabras clave: Desigualdad del ingreso, PBI per cápita, coeficiente de Gini, gestión y políticas públicas, capital y trabajo, equidad, inclusión, globalización, políticas neoliberales

\section{ABSTRACT}

Traditional and marked difference between the rich North and the poor countries of the south, from about 1990 is disappearing more and more. That marked divergence between the increasingly rich and getting poorer countries, which began with the industrial revolution and colonialism in the early nineteenth century, is being reversed by a new convergence.

Today is producing a structural change in the dynamics of the global economy, this new addition to the fast convergence behavior of the rate of economic growth per capita in emerging and developing countries is higher than in developed countries and tends to homogenize the global per capita growth, but these countries have been able not unlink, strong cyclic correlation trend around and have not been able to isolate the effects of the increasing divergence in inequality in the distribution of income between rich and poor.

The 85 richest people in the world now own more than half of the available wealth inequality in income distribution is diverging, harnessing and perpetuating an unprecedented rate worldwide (even though the overall average income is converging), leaving a growing it is «morally wrong» is also shown to slow the rate of growth of countries printing. 


\section{Jorge Barrera Herrera}

Although in this article we focus on the overall income inequality, this is often associated with other inequalities also affect globally and could eventually be even more important (lack of access to food, water, health, education, etc.).

Capital ownership is more concentrated than the distribution of income; $10 \%$ of the population of the OECD have more than 50\% ownership, on the other hand $50 \%$ of the population has no property, but if you are in debt.

Keywords: income inequality, GDP per capita, Gini coefficient, management and public policy, capital and labor, equity, inclusion, globalization, neoliberal policies

\section{Hechos estilizados}

El 1\% de los ciudadanos más ricos de la mayoría de países de la Organización para la Cooperación y el Desarrollo Económico (OCDE) acumulan cada vez más un porcentaje mayor de la renta total. En las últimas tres décadas la desigualdad en el ingreso ha aumentado incluso en países tradicionalmente equitativos como: Suecia, Noruega, Finlandia.

El aumento de las desigualdades en los países avanzados resulta ser menor en comparación con la brecha de la desigualdad entre los países desarrollados y las economías avanzadas.

Según la Unicef, habitamos en un planeta en el que el quintil superior controla más del $80 \%$ del ingreso global, mientras que el quintil del extremo inferior solo controla el $1 \%$ del ingreso global, lo que revela un mundo de una profunda disparidad en el ingreso.

Hay suficiente evidencia que a pesar de los reconocidos beneficios de la desigualdad en premiar el trabajo y la innovación, la desigualdad sin control o excesiva debilitan el crecimiento, por otro lado la igualdad extrema como la redistribución encima de cierto nivel también sería destructivo para el crecimiento.

La última crisis no ha afectado de la misma forma a los acomodados o ricos, quienes aumentaron su riqueza en el 2010 un 4\%, mientras que el 90\% de la población sus ingresos se estancaron. 


\section{Pensamiento Crítico Vol. 19. No 2}

El 20\% de la población mundial controla el 70\% de los ingresos mundiales (Unicef)

Mientras el patrimonio de los 1125 billonarios del mundo crece, el bienestar del resto de la población disminuye. Se está reforzando la concentración de la riqueza: el 1\% de los hogares más ricos posee el $35 \%$ de la riqueza mundial, mientras que el $0.01 \%$ de la población más rica acumula 21 billones dólares (un quinto de la riqueza del planeta).

El coeficiente de Gini, utilizado comúnmente para medir la desigualdad, que varía de cero (0) a uno (1), donde cero es la máxima igualdad, no sirve adecuadamente para poder entender a profundidad las desigualdades de los ingresos entre la población.

El informe de Social Watch refiere que la lucha contra la pobreza va muy por detrás de los avances en otras áreas. "El comercio mundial y el ingreso promedio per cápita mundial crecieron más rápidamente en la primera década del siglo XXI que en la década anterior, pero los avances contra la pobreza se ralentizaron. La brecha se amplió por la desigual distribución de los beneficios de la propiedad".

Entre los años 1990 y 2010, según Social Watch, la evidencia nos demuestra que a pesar de aumentar cinco veces nuestras exportaciones mundiales, pasando de $781 \mathrm{mil}$ millones de dólares a 3.7 billones de dólares y en donde más que duplicaron los ingresos del habitante promedio pasando de \$4,079 a \$ 9,116 por año, pero el promedio mundial del índice de indicadores sociales fundamentales en ese mismo período solo creció el $10 \%$ (de $79.3 \%$ a $87.1 \%$ ).

Según la Oficina del Presupuesto del Congreso de los Estados Unidos, en el 2007 las fuentes de ingresos de mercado eran menos uniformemente distribuidas que 1979 y en ese mismo período las fuentes más concentradas de ingresos crecieron más rápido que las fuentes menos concentradas, además establece que en el 2007 los impuestos y transferencias federales redujeron la dispersión de los ingresos en $20 \%$, pero su efecto igualador fue mayor en 1979.

Según informe de la Unicef, en 64 países de los que se dispone de datos confiables, el número de países con tasas de empleo (empleo/población) en descenso era el doble del que tenía en ascenso a mitad del año 2010. 


\section{Jorge Barrera Herrera}

La OIT (2010) estimó que el $40 \%$ de quienes buscaban trabajo no lo encontraron durante más de un año en una muestra de 35 países y más de cuatro millones habían dejado de buscar trabajo a finales del 2009 en gran parte por la desmoralización y el fracaso de los gobiernos en generar trabajo.

Los jóvenes se han visto desproporcionalmente afectados por el desempleo, según la OIT, el paro juvenil ha crecido en casi ocho millones de personas a nivel global desde el comienzo de la crisis del 2007, siendo más del doble que el de la población activa, esto sin mencionar el notorio descenso de la participación de los jóvenes en el mercado de trabajo y la creciente precariedad y crecimiento del sector informal en el empleo juvenil (OIT 2010), además de las dificultades de las mujeres jóvenes para encontrar trabajo.

Estudios de la Cepal sobre Argentina, Brasil, Chile y Uruguay indican que la mejora del salario mínimo se ha traducido en una caída de la desigualdad, sin afectar negativamente al empleo.

Según Milanovic (2009), utilizando el índice de Gini estableció que la desigualdad global del ingreso ha estado aumentando desde 1820 hasta nuestros días.

\begin{tabular}{cc}
\hline Año & Gini \\
\hline 1820 & 43.0 \\
1850 & 53.2 \\
1870 & 56.0 \\
1913 & 61.0 \\
1929 & 61.6 \\
1950 & 64.0 \\
1960 & 63.5 \\
1980 & 65.7 \\
2002 & 70.7 \\
\hline
\end{tabular}

También si observamos la desigualdad con índice de Gini pero basada en la riqueza, esta también ha estado aumentando 
Pensamiento Crítico Vol. 19. № 2

\begin{tabular}{lccc}
\hline $\begin{array}{l}\text { Desigualdad de la riqueza en } \\
\text { países seleccionados País }\end{array}$ & Gini riqueza (2000) & Gini ingreso & Año \\
\hline Argentina & 74.0 & 50.1 & 2005 \\
Australia & 62.2 & 31.2 & 2003 \\
Bangladesh & 65.8 & 33.5 & 1996 \\
Brasil & 78.3 & 56.6 & 2004 \\
Canadá & 66.3 & 31.5 & 2000 \\
China & 55.0 & 44.9 & 2003 \\
Francia & 73.0 & 27.8 & 2000 \\
Alemania & 67.1 & 31.1 & 2004 \\
India & 66.9 & 36.5 & 1997 \\
Indonesia & 76.3 & 39.6 & 1996 \\
Italia & 60.9 & 33.3 & 2000 \\
Japón & 54.7 & 31.9 & 1998 \\
Corea del Sur & 57.9 & 37.2 & 1998 \\
México & 74.8 & 49.9 & 2004 \\
Nigeria & 73.5 & 52.2 & 1996 \\
Pakistán & 69.7 & 39.8 & 1996 \\
España & 56.5 & 33.6 & 2000 \\
Taiwán & 65.4 & 33.9 & 2003 \\
Tailandia & 70.9 & 42.7 & 2001 \\
Estados Unidos & 80.1 & 46.4 & 2004 \\
Vietnam & 68.0 & 37.3 & 1998 \\
\hline
\end{tabular}

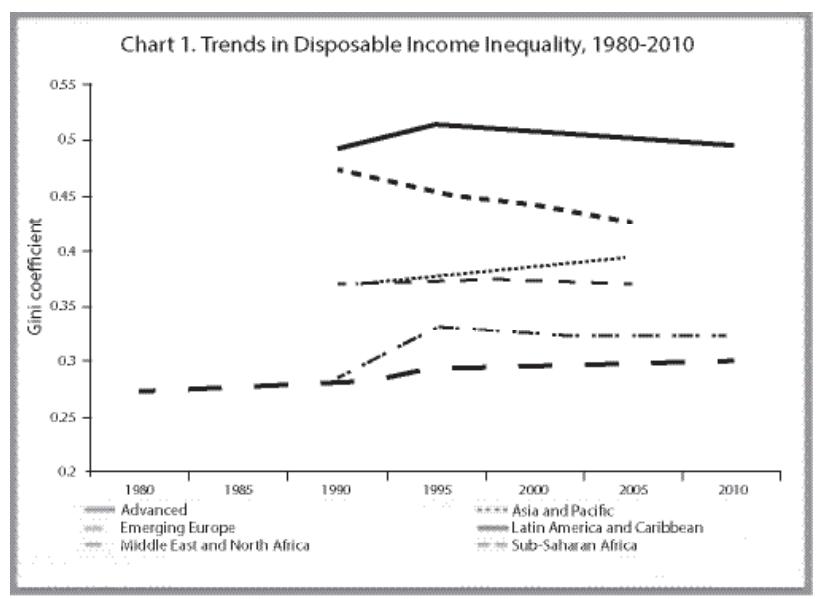




\section{Jorge Barrera Herrera}

\section{Causas}

\section{Convergencia en las tasas de crecimiento per cápita de los países}

Son varios los factores que motivan esta nueva convergencia:

Es con la globalización, la apertura de los mercados, los adelantos en las comunicaciones, el fomento y facilidad del comercio y de los flujos de capitales a través de la inversión extranjera directa, por la veloz expansión de su clase media, que los países antes rezagados han podido acceder y adoptar nueva tecnología y conocimientos técnicos.

El efecto marginal de la transición demográfica, por la reciente importancia del control del crecimiento poblacional en los países rezagados, promovió una mayor intensidad del capital acelerando el efecto del crecimiento per cápita, mientras que paralelamente, en los países avanzados, con larga historia de control poblacional la proporción de personas de tercera edad creció significativamente.

Mayor proporción de los ingresos es invertido en los países rezagados de mano de obra barata, de necesidades básicas todavía mayoritariamente insatisfechas, por las facilidades y altos rendimientos que generan; esto no solo incrementa la productividad de la mano de obra, sino también impulsa mayor productividad de los factores incorporando conocimientos y técnicas de producción facilitando la transición desde sectores de escasa productividad como la agricultura, la minería, hacia sectores de gran productividad y de mayor valor agregado, como la manufactura.

Las presiones fiscales, el sobreendeudamiento, el deterioro de sus monedas, haber cubierto mayoritariamente sus necesidades básicas y el mayor gasto en los países avanzados están en contraposición a la mayor estabilidad macroeconómica y apreciación de sus monedas, satisfacer todavía necesidades básicas en la población en los países emergentes y en desarrollo más ahorradores que los financian, lo cual facilita también la nueva convergencia. 


\section{Pensamiento Crítico Vol. 19. № 2}

\section{Divergencia en la distribución del ingreso global}

Son varias las causas que motivan esta divergencia:

La necesaria, tradicional y equilibrada mutua dependencia entre empleado y empleador, entre las fuerzas del capital y las fuerzas del trabajo, entre las posibilidades y las necesidades, entre el valor de cambio y el valor de uso, está yendo en divergencia por la ruptura del capital de forma unilateral. El Estado en los años 70 desreguló, privatizó y subcontrató obligaciones tradicionales con sus ciudadanos (neoliberalismo y globalización); los dueños del capital vieron que ya no era de su interés ocuparse de los demás, estableciendo una nueva relación de desequilibrios, desarmando la economía del bienestar imperante.

Según el economista francés Picketty, una gran minoría propietaria del capital históricamente tienden a establecer que la tasa de retorno del capital $(\mathrm{R})$ siempre debe ser mayor que la tasa de crecimiento económica de los países (g), lo que de manera indefectible generará que los pocos poseedores de la propiedad del capital tengan cada vez más ganancias o beneficios y por lo tanto más poder, esto se logrará dando salarios bajos por la siempre presente cantidad de desempleados permanentes, alentando y perpetuando la desigualdad en los ingresos. Buffet, uno de los hombres más ricos del mundo cuando se le preguntó sobre la última crisis económica, dijo: "por supuesto que es lucha de clases y mi clase, la de los ricos, gana".

Por tanto, un mayor crecimiento económico, donde $\mathrm{R}>\mathrm{g}$, implicará un incremento de las desigualdades en la distribución de los ingresos, generando incentivos perversos de concentración del capital, estableciendo una mayor influencia hacia la dominación de la riqueza heredada. La idea de que el libre mercado garantiza la competencia y la imparcialidad resulta cada vez menos evidente, la desigualdad en la propiedad no lo resuelve el mercado ni el crecimiento en sí, por ser un problema cultural y político requiere soluciones también en este campo.

Según Picketty, la solidez de la relación $\mathrm{R}>\mathrm{g}$ no depende mayormente de factores fuera del mercado, de las habilidades y de la educación, sino más bien depende de la influencia de los lobbies políticos de los ricos en su efecto sobre las políticas fiscales y de las condiciones laborales (pago de impuestos, focalización y resultados eficientes del gasto público). 


\section{Jorge Barrera Herrera}

Cuanto mayor es el poder de la clase capitalista (inexistencia de los sindicatos), mayor es la dispersión salarial; el argumento de eficiencia económica y de aumento de la productividad no son suficientemente convincentes para explicar la creciente desigualdad de los ingresos.

Existe un divorcio entre poder y política; antes de los años 70 esto estaba junto en manos del Estado soberano, ahora está relación es diferente por las nuevas fuerzas de la globalización, estableciendo una relación creciente de interdependencia que se está todavía construyendo.

Se asume que por la globalización y con la movilidad de capitales que se desplaza a países de bajos salarios para abaratar la producción de los productos en un contexto político facilitador se produce la desigualdad de los ingresos, pero esta propuesta tradicional no explicaría totalmente cómo se generan los altos sueldos de los súper gerentes.

Kuznets establece que, en la etapa inicial del crecimiento económico de los países, la desigualdad en la distribución del ingreso tiende a crecer, para luego en el mediano y largo plazo lograr estabilizarse y comenzar a disminuir en la medida que aumentan los ingresos; la evidencia actual de perpetuación y crecimiento de las desigualdades, independientemente de los ciclos o etapas, estarían desvirtuando esta afirmación.

El aumento relativo de la población mundial de edad avanzada, por el mejoramiento de la calidad de vida y mejores beneficios asistenciales sanitarios y disminución del crecimiento demográfico, está motivando una desaceleración de la productividad con el dominio cada vez mayor de la riqueza heredada, favoreciendo la desigualdad del ingreso.

En algunos países, especialmente en los Estados Unidos, la política fiscal se ha vuelto regresiva, es decir que en proporción a su renta, los ricos pagan cada vez menos impuestos que los pobres. 
Pensamiento Crítico Vol. 19. № 2
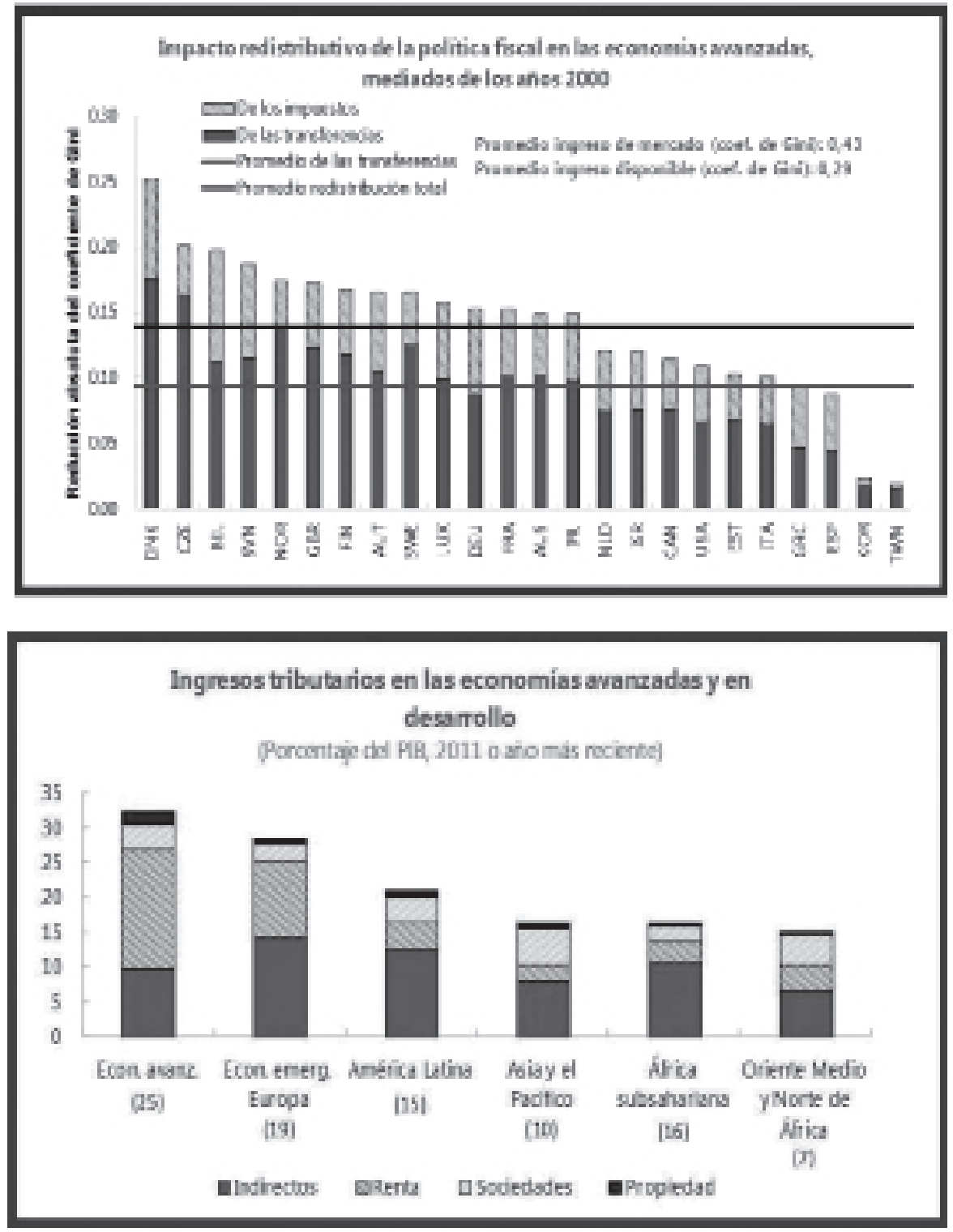


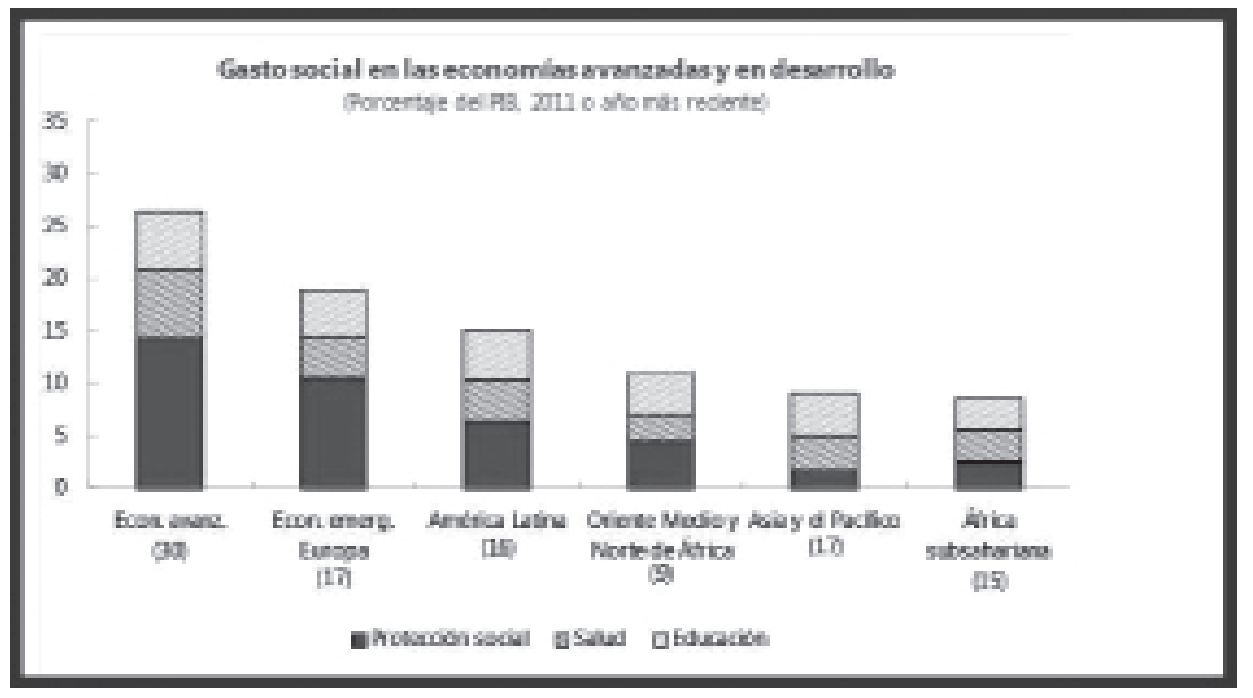

\section{Consecuencias negativas de la desigualdad}

1. Ralentiza el crecimiento económico

Alesina y Rodrick (1994), Bourguignom (2004) y Birdsall (2005) han demostrado que países en desarrollo con alta desigualdad tienden a crecer más lentamente.

2. Genera problemas sociales y de salud

Wilkinson y Pickett (2010) han demostrado que los individuos en sociedades más equitativas disfrutan de mejor salud, viven más, tienen menos probabilidades de sufrir enfermedades mentales, etc., en comparación con sociedades menos equitativas.

3. Produce inestabilidad política

Kaufman (2010), utilizando indicadores mundiales de gobernanza (WGI), estableció que las sociedades desiguales en general son mucho más propensas a la inestabilidad política, lo que incluye terrorismo. 


\section{Pensamiento Crítico Vol. 19. No 2}

4. Condena a desigualdades más graves, especialmente entre la infancia Un estudio de Unicef (2010) establece que en relación para los niños, existe una fuerte relación negativa entre una mayor desigualdad de ingreso y niveles más bajos de educación y de desigualdades en la salud.

\section{Medidas correctivas}

La Unicef señala que, a pesar de haberse producido algunas mejoras en la disminución de la desigualdad, estos avances son demasiado lentos. Según data histórica, para que los mil millones de personas en los quintiles de abajo, viviendo en extrema pobreza, mejoren su proporción de ingreso mundial en $0.18 \%$, se necesitarían 17 años. Por tanto, en la actualidad, manteniendo esta velocidad, se necesitarían más de 8 siglos para que esos mil millones de más pobres del mundo alcancen el $10 \%$ del ingreso global.

Las principales medidas correctivas son las políticas de transferencias, del gasto público y los impuestos, que se logren establecer entre el poder político, el capital y los trabajadores.

El impacto distributivo de la política fiscal ha disminuido desde 1990, la desigualdad creció de 1980 al 2000 dos veces más que la redistribución. Se debe evaluar el aumento de los impuestos y del gasto público, siempre en forma conjunta.

Paul Krugman decía que "la productividad no lo es todo, pero en el largo plazo es casi todo". Además, en ocasiones la mano invisible del mercado no produce resultados eficientes, por las normales fallas del mercado, por eso es necesario tener un Estado que tenga un plan nacional de diversificación de la producción, intervenir solo cuando hay fallas de mercado y cuando el instrumento de política que la corrige genera beneficios mayores a sus costos, con el fin de hacer menos atractiva las actividades rentistas que no crean valor real en la economía y que protejan e incentiven las inversiones en los espontáneos y audaces procesos de autodescubrimiento empresarial respecto de actividades donde el país es más competitivo. Acciones de este tipo favorecen las igualdades, hacen más consistente el crecimiento, se logra mayor productividad de las

empresas y hacen más eficiente la transferencia de recursos entre sectores en el marco de un fácil acceso al financiamiento internacional. 


\section{Jorge Barrera Herrera}

\section{Necesidad de llevar la equidad a la agenda del desarrollo}

En vista de la importancia de aliviar la pobreza y miseria creciente de una gran mayoría global poblacional, es necesario centrar la atención en el hecho de que los efectos de las políticas que se decidan en el mundo recaen principalmente en la minoría de más concentración de ricos, por lo que se debería situar a la equidad en el centro de la agenda del desarrollo y preguntarnos para quién es el desarrollo.

Según informe del Banco Mundial (2006), la equidad no es algo opuesto al crecimiento; de hecho estimula el consumo, eleva la productividad y sustenta el crecimiento. Por tanto, encontrar la armonía, el equilibrio, la combinación adecuada de políticas para alcanzar crecimiento con equidad será la clave del desarrollo humano del futuro (Kanbur y Lusting 1998, Van der Heven et. al. 2001).

\section{Fomentar y promover el empleo, la dependencia a los precios crecientes de los alimentos, la austeridad fiscal y la importancia de la inversión en la primera infancia}

El vínculo entre el desempleo y el precio creciente de los alimentos en las familias más pobres con la desigualdad es cada vez más evidente y notorio. El Banco Asiático de Desarrollo (BAD 2008) calcula que un aumento del $20 \%$ del precio nominal de los alimentos conduce a un aumento del $1 \%$ en el coeficiente de Gini.

En términos de desigualdad, la reducción del gasto público en educación básica, sanidad y programas de seguridad social van asociados a caídas en los ingresos de los grupos más pobres.

Los actuales comportamientos del empleo, precios de los alimentos y el combustible, y gastos público parecen indicar un aumento de las desigualdades, por lo que es clara la necesidad de revertir esta tendencia aplicando políticas de inclusión que garanticen recuperación para todos, en especial de los hogares más excluidos.

Varios estudios de investigación señalan que los retornos de la inversión en educación son mayores cuando esas habilidades son formadas más temprano en el 


\section{Pensamiento Crítico Vol. 19. No 2}

ciclo de vida, además una de las formas más eficientes de eliminar las desigualdades en la distribución del ingreso es invertir en la primera infancia, especialmente en los niños desfavorecidos más pequeños, rompiendo el ciclo de la "pobreza heredada". Los beneficios de invertir en la primera infancia: "provee la equidad y la justicia social y al mismo tiempo promueve la productividad en la economía y en la sociedad en general"

\section{Conclusión}

Es necesario que la estructura de gobierno de las instituciones internacionales, como el Fondo Monetario Internacional (FMI), El Banco Mundial (BM), la Organización Mundial del Comercio (OMC), la Organización Mundial del Trabajo (OIT), etc., refleje el nuevo peso de las economías emergentes y en desarrollo.

El ciclo económico global, por sus efecto contagio, al igual que las divergencias en la distribución del ingreso, requiere de mayores esfuerzos de coordinación de políticas macroeconómicas y migratorias globales.

Para lograr la erradicación simultánea de la pobreza y la reducción significativa de las desigualdades y la exclusión, y promover el desarrollo humano y sostenible en los países emergentes y en desarrollo, según la PNUD, requiere de una profunda valoración y aplicación de los conceptos de vulnerabilidad y resiliencia. Los avances en el crecimiento y principalmente en el desarrollo solo podrán ser equitativos y sostenibles si las vulnerabilidades se enfrentan con eficacia y si todas los ciudadanos, en especial los menos favorecidos y empoderados, disfrutan de la oportunidad de participar en esos avances.

El mundo cada vez deberá ser más multipolar e interdependiente y el acceso a los mercados seguirá siendo la gran oportunidad de crecimiento para los países rezagados y alcanzar el bienestar de los países avanzados. Aprovechar estas ventajas dependerá de que la cooperación internacional logre coordinar mejores políticas macroeconómicas nacionales, teniendo en cuenta sus efectos de contagio, promoviendo la equidad y el equilibrio en la distribución del ingreso.

Dado el gran potencial todavía por crecer en los países rezagados y a pesar de que el crecimiento de la productividad marginal es decreciente, son muchos los sectores que 


\section{Jorge Barrera Herrera}

todavía faltan incorporarse a la nueva tecnología e innovación, por lo que la tendencia a la convergencia de los ingresos per cápita global se mantendrá.

Considerando la gravedad y persistencia del desempleo global, es probable que la desigualdad en las ganancias continúe, de no realizarse las acciones y políticas adecuadas para modificar y cambiar esa tendencia.

La sostenibilidad de cualquier esfuerzo en mejorar la distribución del ingreso global pasa necesariamente por establecer medidas que permitan compartir ampliamente los beneficios, es decir darle mayor importancia a lograr ajustes y crecimiento con equidad en la búsqueda de la armonía y equilibrios.

El crecimiento económico basado solamente en las exportaciones de los recursos naturales y en el gasto público genera un olvido de la productividad, favorece la desigualdad en la distribución del ingreso y deteriora la sustentabilidad de las actividades.

Hay que buscar un balance en las políticas públicas del gasto entre la asignación a proyectos selectivos (desarrollo de clústeres) y a los aplicados de manera neutra (protección de la propiedad intelectual, subsidio crédito tributario al esfuerzo de innovación, emprendimiento dinámico imparcial) con especial énfasis en los programas de emprendimiento dinámico en los que no hay un mercado privado para las primeras etapas de financiamiento.

Es necesario cambiar la fiscalidad del trabajo frente al capital, de modo de lograr el equilibrio adecuado entre los beneficios y los salarios; de lo contrario, el consumo será insuficiente y, por tanto, no habrá crecimiento económico.

Es necesario hacer los mejores esfuerzos para promover aquellas políticas de comercio y finanzas internacionales y de protección social, tener un mayor reconocimiento de los derechos individuales y colectivos y tener en consideración los problemas de representación en el ámbito político, que tengan las mayores consecuencias positivas sobre el desarrollo nacional inclusivo y beneficien a las grandes mayorías de un país dentro de la aldea global. 


\section{Pensamiento Crítico Vol. 19. No 2}

Con la participación de las fuerzas políticas, es necesario lograr construir un nuevo estatus entre el capital y el trabajo, donde el ser humano sea lo fundamental, teniendo en cuenta que los recursos son finitos, donde es necesario cuidar el medio ambiente de la aldea global y que todos somos interdependientes en la identificación, utilización y valorización del bien común para alcanzar un sostenido bienestar que nos asegure la supervivencia.

"Al abordar las vulnerabilidades, todas las personas podrán compartir los progresos en materia de desarrollo, y el desarrollo humano se volverá cada vez más equitativo y sostenible". Helen Clark, administradora del Programa de las Naciones Unidas para el Desarrollo.

"La resiliencia humana consiste en eliminar las barreras que impiden que las personas puedan actuar con libertad. Consiste también en permitir que los grupos más desfavorecidos y excluidos puedan expresar sus preocupaciones, sean escuchados y sean agentes activos en la definición de su propio destino". Khalid Malik, autor principal del informe, capítulo 1.

"A pesar de los importantes y variados progresos alcanzados, existen todavía personas y grupos más vulnerables, siendo los discapacitados uno de los peor parados. Las Naciones Unidas estiman que más de mil millones de personas viven con algún tipo de discapacidad y estas se encuentran, de forma desproporcionada, entre las personas más pobres del mundo". Profesor Stephen Hawking, capítulo 1.

"Si la oposición constructiva es imposible, quienes no se conformen con dedicar su vida a pagar las deudas contraídas por otro, no tienen otra salida que la opción destructiva" (Buying Time).

\section{Referencias bibliográficas}

Alvaredo F. (2011). "A Note on the Relationship between Top Income Shares and the Gini Coefficient," Economics Letters, Vol. 110(3), pp. 274-77.

Berg, A., J.D. Ostry, and J. Zettelmeyer (2012). "What Makes Growth Sustained?" Journal of Development Economics, Vol. 98(2), pp. 149-66. 


\section{Jorge Barrera Herrera}

Lustig, N., C. Pessino, and J. Scott (2013). "The Impact of Taxes and Social Spending on Inequality and Poverty in Argentina, Bolivia, Brazil, Mexico, Peru and Uruguay: An Overview," Working Paper No. 1313, Tulane University, Department of Economics.

Stiglitz, J. (2012). The Price of Inequality: How Today's Divided Society Endangers Our Future, W. W. Norton \& Company.

Thewissen, S.H. (2013). "Is it the Income Distribution or Redistribution that Affects Growth?" Socio-Economic Review, forthcoming (advance online publication october 2013).

International Monetary Fund (IMF) (2014). Redistribution, Inequality, and Growth. Prepared by Jonathan D. Ostry, Andrew Berg, Charalambos G. Tsangarides. 